\title{
ON QUANTIZATION AND COMMUNICATION \\ TOPOLOGIES IN MULTI-VEHICLE RENDEZVOUS $^{1}$
}

\author{
Karl Henrik Johansson* Alberto Speranzon *,2 \\ Sandro Zampieri ${ }^{* *}$ \\ * Department of Signals, Sensors and Systems \\ Royal Institute of Technology \\ Osquldas väg 10, 100-44 Stockholm, Sweden \\ $\{k a l l e j$, albspe\}@s3.kth.se \\ ** Dipartimento di Elettronica e Informatica \\ Universitá di Padova \\ via Gradenigo, 6/A, I-35131 Padova, Italy \\ zampi@dei.unipd.it
}

\begin{abstract}
A rendezvous problem for a team of autonomous vehicles that is communicating through quantized channels is considered. Communication topologies and feedback control law are presented that solves the rendezvous problem in the sense that a meeting point for the vehicles is practically stabilized. In particular, it is shown that uniform quantizers can sometimes be replaced by logarithmic quantizers and thus reduce the need for communication bandwidth.

Copyright (C)2005 IFAC
\end{abstract}

Keywords: Control under limited communication; Quantized control;

Multi-vehicle coordination; Distributed control; Hybrid systems.

\section{INTRODUCTION}

Interplay between coordination and communication is important in many multi-vehicle systems, e.g., car platoons on automated highways (Varaiya, 1993), formations of autonomous underwater vehicles (de Sousa and Pereira, 2002), and multi-robot search-and-rescue missions

(Speranzon and Johansson, 2003). Constrained communication between vehicles suggest the deployment of distributed (local) control strategies (Lin et al., 2003; Saber and Murray, 2003). In many cases not only the communication topology is important, however, but also the amount of

\footnotetext{
1 This research is supported by the European project RECSYS, IST-2001-32515.

2 Corresponding author.
}

data being transmitted. Therefore, in this paper we study multi-vehicle control under quantized communication. The problem is related to the stabilization of linear plants with quantized control, which has recently been extensively studied, see (Fagnani and Zampieri, 2003) and references therein.

The main contribution of this paper is to illustrates how communication topologies based on uniformly and logarithmically quantized communication influence the solution to a multi-vehicle rendezvous problem. A team of autonomous vehicles with only local position information is to meet under minimum communication capabilities. We prove the existence of several classes of solutions to this rendezvous problem. In particular, we emphasize that uniform quantizers can sometimes 
be replaced by logarithmic quantizers and thus reduce the need for communication bandwidth.

The outline of the paper is as follows. The rendezvous problem is defined in Section 2. Illustrative two-vehicle cases are studied in detail in Section 3. Teams of three and more vehicles are then considered in Section 4. Convergence properties are investigated through simulations in Section 5 . Some conclusions are given in Section 6 .

\section{PROBLEM FORMULATION}

Consider $n \geq 2$ vehicles moving in a plane, with dynamics described by the discrete-time system

$$
\begin{aligned}
& \mathbf{x}^{+}=\mathbf{x}+\mathbf{u} \\
& \mathbf{y}^{+}=\mathbf{y}+\mathbf{v}
\end{aligned}
$$

where $\mathbf{x}=\left(x_{1}, \ldots, x_{n}\right)^{T} \in \mathcal{X} \subset \mathbb{R}^{n}$ and $\mathbf{y}=$ $\left(y_{1}, \ldots, y_{n}\right)^{T} \in \mathcal{Y} \subset \mathbb{R}^{n}$, so that $\left(x_{i}, y_{i}\right)$ denotes the position of vehicle $i$ with respect to a fixed coordinate system. Let $\mathcal{U} \subset \mathbb{R}^{n}$ and $\mathcal{V} \subset \mathbb{R}^{n}$ denote the set of control values. The controls $\mathbf{u}=\left(u_{1}, \ldots, u_{n}\right)$ and $\mathbf{v}=\left(v_{1}, \ldots, v_{n}\right)$ we are considering are feedback maps from the corresponding state-space $\mathcal{X}$ and $\mathcal{Y}$, respectively. Since the control of the $x$ - and $y$-coordinates are independent, we only consider $x$ in the sequel.

\subsection{Quantized communication}

We further restrict the communication by imposing that communicated data are quantized. In particular, uniform and logarithmic quantizations are considered. Recall the following definitions of scalar uniform and logarithmic quantizers.

Definition 1. Let $\delta_{u}$ be a positive parameter. A uniform quantizer is a map $q_{u}: \mathbb{R} \rightarrow \mathbb{R}$ such that

$$
q_{\mathrm{u}}(x)=\delta_{u}\left\lfloor\frac{x}{\delta_{u}}\right\rfloor .
$$

Notice that the error due to the quantization of a variable $x$ is bounded by $\delta_{u}$ i.e.,

$$
\left|q_{u}(x)-x\right| \leq \delta_{u}
$$

Definition 2. Let $\delta_{\ell}$ be a positive parameter. A logarithmic quantizer is a map $q_{\ell}: \mathbb{R} \rightarrow \mathbb{R}$ such that

$$
q_{\ell}(x)=\exp \left(q_{u}(\ln x)\right)
$$

The quantization error for a logarithmic quantizer is bounded as

$$
\left|q_{l}(x)-x\right| \leq \delta_{\ell}|x| .
$$

Notice that the parameter $\delta_{\ell}$ depends on $\delta_{u}$ through the following expression

$$
\delta_{\ell}=1-e^{-\delta_{u}}
$$

We are interested in how communication topology and quantization of transmitted data influence the performance of the multi-vehicle system. Therefore, we consider classes of maps $\Psi_{\delta}$ composed of various configurations of uniform and logarithmic quantizers. For simplicity, we suppose that all quantizers are parameterized in a single quantization parameter $\delta$.

\subsection{Rendezvous}

We are interested in the convergence to a multivehicle formation under quantized communication topology. Especially, we pose the following rendezvous problem.

Definition 3. A feedback law $(u)=g(\mathbf{x})$ solves the rendezvous problem if for all initial states $\mathbf{x}_{0} \in \mathcal{X}$, and for all $\epsilon>0$, there exists $\delta=\delta(\epsilon)$ such that

$$
x_{i}-x_{j} \rightarrow \mathcal{B}_{\epsilon}
$$

with $i<j$ and $i, j=1, \ldots, n$.

A solution to the rendezvous problem is able to make the vehicles converge arbitrarily close to a meeting point. Note that the meeting point is not pre-specified, but is only restricted to be close to the hyperplane $x_{1}=\cdots=x_{n}$.

If the communication is not quantized, then the rendezvous problem is readily solved also in the case with no communication. In this case, a decentralized deadbeat controller provides a solution. We consider such a solution trivial, since it enforces the vehicles to meet in the origin. It is instead desirable to have the meeting point close to the initial position of the vehicles. In next section, we present a linear quadratic control problem for the two-vehicle system that address this problem and that also suggest a natural extension to the quantized case.

\section{TWO-VEHICLES RENDEZVOUS}

Let $z=x_{1}-x_{2}$ be the output of the system (1) when $n=2$. In order to avoid aggressive solution such as the dead-beat control we need to penalize the control input. Thus we consider an LQ problem with cost

$$
\begin{aligned}
J\left(u_{1}, u_{2}\right) & =\sum_{t=0}^{\infty} z^{2}+u_{1}^{2}+u_{2}^{2} \\
& =\sum_{t=0}^{\infty}\left(x_{1}-x_{2}\right)^{2}+u_{1}^{2}+u_{2}^{2} .
\end{aligned}
$$

The resulting state feedback $\mathbf{u}=\mathbf{K} \mathbf{x}$ gives the closed-loop system

$$
\begin{aligned}
& x_{1}^{+}=x_{1}-k\left(x_{1}-x_{2}\right) \\
& x_{2}^{+}=x_{2}-k\left(x_{2}-x_{1}\right),
\end{aligned}
$$


with $k=1 /(1+\sqrt{3})$. It corresponds to

$$
z^{+}=(1-2 k) z
$$

which is asymptotically stable (in general for $0 \leq$ $k \leq 1$ ), for the optimal choice of $k$. This means the difference $x_{1}-x_{2}$ tends to zero asymptotically. Note that (5) it is only stable, thus the two vehicles, in general, will rendezvous to a point different from the origin. The linear feedback $\mathbf{K}$ computed above is used to design controllers when the communication topology is composed of various configurations of uniform and logarithmic quantizers.

\subsection{Uniform-uniform quantization}

Proposition 4. The feedback

$$
\begin{aligned}
& u_{1}=-k\left(x_{1}-q_{u}\left(x_{2}\right)\right) \\
& u_{2}=-k\left(x_{2}-q_{u}\left(x_{1}\right)\right)
\end{aligned}
$$

solve the rendezvous problem

Proof. Let us consider the difference $z=x_{1}-$ $x_{2}$. We introduce the following Lyapunov function $\mathcal{V}(z)=|z|$. Thus the increment $\Delta \mathcal{V}(z)=\mathcal{V}\left(z^{+}\right)-$ $\mathcal{V}(z)=\left|z^{+}\right|-|z|$ is such that

$$
\begin{aligned}
\Delta \mathcal{V}(z) & =\left|z-2 k z-q_{u}\left(x_{2}\right)+q_{u}\left(x_{1}\right)\right|-|z| \\
& \leq-2 k|z|+2 k \delta
\end{aligned}
$$

where we have used (3). Hence $\Delta \mathcal{V}(z)<0$ if $|z|>$ $\delta$. If we choose $\delta=\epsilon$, then $z$ will asymptotically converge to $\mathcal{B}_{\epsilon}$. Thus the feedback (6) solves the rendezvous problem.

Notice that instead of posing a deterministic LQ problem, we can consider a stochastic LQ problem where the quantization error of the uniform quantizer is approximated by additive white noise, namely

$$
q_{u}(x)=x+e
$$

and where $e$ is white noise uniformly distributed in $[-\delta, \delta]$ and $E e^{2}=\delta^{2} / 12$. This approach yields the same feedback control as above.

\subsection{Uniform-logarithmic quantization}

Proposition 5. The feedback

$$
\begin{aligned}
& u_{1}=-k q_{\ell}\left(q_{u}\left(x_{1}\right)-x_{2}\right) \\
& u_{2}=-k\left(x_{2}-q_{u}\left(x_{1}\right)\right)
\end{aligned}
$$

solve the rendezvous problem

Proof. Let us consider the difference $z=x_{1}-x_{2}$. As in the proof of Proposition 4,

$$
\begin{aligned}
\Delta \mathcal{V}(z) & =\left|z-k\left(q_{\ell}\left(q_{u}\left(x_{1}\right)-x_{2}\right)-x_{2}+q_{u}\left(x_{1}\right)\right)\right|-|z| \\
& \leq-2 k|z|+k|z| \delta+2 k \delta+k \delta^{2}
\end{aligned}
$$

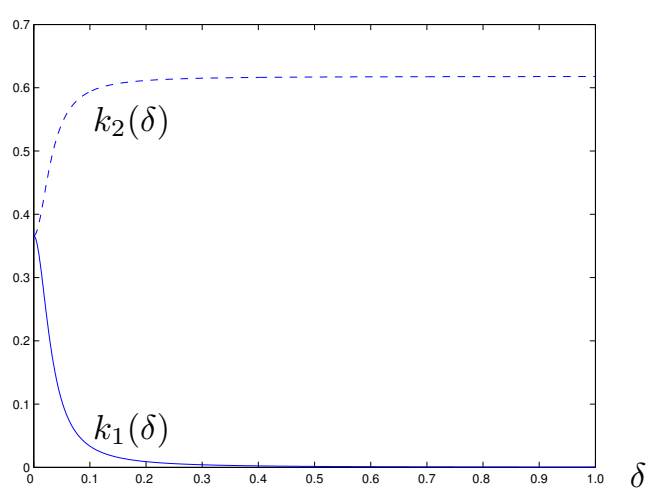

Fig. 1. For large quantization step $\delta$ the value of $k_{1}(\delta)$ tends to zero while the value of $k_{2}(\delta)$ tends to the value $k_{2}(1)$, obtained solving an optimal control problem with $n=1$.

where we used (4). $\Delta \mathcal{V}(z)<0$ if

$$
|z|>\frac{2 \delta(1+\delta)}{2-\delta}
$$

If we choose $\delta=-1 / 2-\epsilon / 4-\sqrt{4+20 \epsilon+\epsilon^{2}} / 4$, $z$ converges asymptotically to $\mathcal{B}_{\epsilon}$. Thus the feedback (7) solves the rendezvous problem.

The effect of the logarithmic quantization can be approximate as multiplicative noise acting on the system. If, for simplicity, we neglect the presence of uniform quantization, then we have

$$
\begin{aligned}
& x_{1}^{+}=x_{1}+k_{1}\left(x_{1}-x_{2}\right)(1+e) \\
& x_{2}^{+}=x_{2}+k_{2}\left(x_{2}-x_{1}\right)
\end{aligned}
$$

where $e$ is white noise uniformly distributed in $[-\delta, \delta]$ and variance $E e^{2}(t)=\delta^{2} / 12$. Let $z=x_{1}-$ $x_{2}$, we consider an optimal control problem with cost

$$
\begin{aligned}
J\left(u_{1}, u_{2}\right) & =E \sum_{t=0}^{\infty} z^{2}+u_{1}^{2}(1+e)+u_{2}^{2} \\
& =E \sum_{t=0}^{\infty} z^{2}+\left(u_{1}, u_{2}\right) \underbrace{\left(\begin{array}{cc}
1+\frac{\delta^{2}}{12} & 0 \\
0 & 1
\end{array}\right)}_{R}\left(\begin{array}{l}
u_{1} \\
u_{2}
\end{array}\right)
\end{aligned}
$$

and dynamics

$$
z^{+}=z+\underbrace{(11)}_{B}\left(\begin{array}{l}
u_{1} \\
u_{2}
\end{array}\right)+u_{1} e .
$$

The feedback law is linear and given by

$$
K=\left(R+B^{T} P B+\Omega(P)\right)^{-1} B^{T} P
$$

where $P$ is the solution of a generalized Riccati difference equation and $\Omega(P)$ is the following matrix

$$
\Omega(P)=\left(\begin{array}{cc}
1+\frac{\delta^{2}}{12} & 0 \\
0 & 0
\end{array}\right),
$$

see (Beghi and D'Alessandro, 1998). The optimal feedback is

$$
\begin{aligned}
& k_{1}(\delta)=k+a \delta^{2}+O\left(\delta^{4}\right) \\
& k_{2}(\delta)=k+b \delta^{2}+O\left(\delta^{4}\right)
\end{aligned}
$$


where $k$ is the same value computed solving the deterministic optimal control problem and $a, b$ are two real values an order of magnitude less than $k$. If we plot $k_{1}(\delta)$ and $k_{2}(\delta)$ we observe that for large value of $\delta$ the gain $k_{1}(\delta)$ tends to zero, see Figure 1, meaning that the first vehicle, whose input depends on the logarithmically quantized value of the relative distance $x_{1}-x 2$, does not move since $x_{1}-x_{2}$ is known with very large error. On the other hand the second vehicle, which rely on a perfect knowledge of the position of the first, applies a large input in order to move towards it (see Figure 1). The value of the gain for $\delta=1$ is

$$
k_{2}(1)=\frac{1+\sqrt{5}}{3+\sqrt{5}}
$$

which the optimal gain static gain when we have a single vehicle.

\subsection{Logarithmic-logarithmic quantization}

An interesting communication configuration is the one where the data is exchanged over logarithmic quantized channel. We assume also here that the uniform quantization can be neglected. For this communication configuration we can show that for a particular choice of $k$ in the interval $0 \leq k \leq 1$ the two vehicles do not rendezvous in average. Also in this case we model the logarithmic quantization as a multiplicative noise.

Proposition 6. Consider feedback laws

$$
\begin{aligned}
& u_{1}=-k q_{\ell}\left(q_{\ell}\left(x_{1}\right)-x_{2}\right) \\
& u_{2}=-k\left(x_{2}-q_{\ell}\left(x_{1}\right)\right)
\end{aligned}
$$

with $k=1 / 2$, then the two vehicle do not rendezvous in average.

Proof. Modeling the quantization as multiplicative noise we have

$$
\begin{aligned}
\mathbf{x}^{+} & =\left(\begin{array}{cc}
1 / 2 & 1 / 2 \\
1 / 2 & 1 / 2
\end{array}\right) \mathbf{x}+e_{1}\left(\begin{array}{cc}
-1 / 2 & 0 \\
1 / 2 & 0
\end{array}\right) \mathbf{x} \\
& +e_{2}\left(\begin{array}{cc}
-1 / 2 & 1 / 2 \\
0 & 0
\end{array}\right) \mathbf{x}+e_{1} e_{2}\left(\begin{array}{cc}
-1 / 2 & 0 \\
0 & 0
\end{array}\right) \mathbf{x}
\end{aligned}
$$

where we assume that $e_{1}$ and $e_{2}$ are random variables uniformly distributed and independent. Let

$$
Q(t)=E\left(x(t) x^{T}(t)\right)=\left(\begin{array}{ll}
Q_{1}(t) & Q_{2}(t) \\
Q_{2}(t) & Q_{3}(t)
\end{array}\right)
$$

then we can study the stability properties of

$$
\begin{aligned}
P(t+1) & =A(\lambda) P(t) \\
E\left(z^{2}\right) & =(1-1) P(t)\left(\begin{array}{c}
1 \\
-1
\end{array}\right)=\underbrace{(1-21)}_{C} P(t)
\end{aligned}
$$

where $P(t)=\left(Q_{1}(t), Q_{2}(t), Q_{3}(t)\right)$ and where we assume $E\left(e_{1}^{2}\right)=E\left(e_{2}^{2}\right)=\lambda$. If we consider

$$
q(z)=\operatorname{det}(z I-A(\lambda))
$$

and we study the polynomial $p(s)=q((1+$ $s) /(1-s))$ we find out with Routh-Hurwitz that the polynomial $p(s)$, and thus $q(z)$, is not stable. Since the system (9) is observable, then we can conclude that system (9) is an unstable system, meaning that the variance increases, and thus the two vehicles are likely to not rendezvous.

\section{N-VEHICLES RENDEZVOUS}

For any pair of vehicles $(i, j), i<j$ we define an output variable $w_{i, j}=x_{i}-x_{j}$. Let $\mathbf{w}$ be a vector collecting all subset of such output variables. Similar to the two vehicles case we consider an optimal control problem with cost function

$$
\begin{aligned}
J(\mathbf{u}) & =\sum_{t=1}^{\infty} \mathbf{w}^{T} \mathbf{w}+\mathbf{u}^{T} \mathbf{u} \\
& =\sum_{t=1}^{\infty} \mathbf{x}^{T} W^{T} W \mathbf{x}+\mathbf{u}^{T} \mathbf{u} .
\end{aligned}
$$

Note the matrix $W^{T} W$ is singular, it is anyway possible to regularize the problem. Consider any subset $\mathbf{z}=\left\{z_{i, j}\right\}$ of $\mathbf{w}$. Let $Z \in \mathbb{R}^{(n-1) \times n}$ such that $\mathbf{z}=Z \mathbf{x}$. Then there exists a matrix $L$ such that

$$
\mathbf{w}=\left(\begin{array}{l}
I \\
L
\end{array}\right) \mathbf{z} .
$$

Minimizing the cost function (10) subject to the dynamics (1) is equivalent to minimizing the cost

$$
J(\mathbf{u})=\sum_{t=1}^{\infty} \mathbf{z}^{T}\left(I+L^{T} L\right) \mathbf{z}+\mathbf{u}^{T} \mathbf{u}
$$

subject to dynamics $\mathbf{z}^{+}=\mathbf{z}+Z \mathbf{u}$. The optimal control law for the system (1) is such that $\mathbf{u}=$ $K Z \mathbf{x}$ with $^{3}$

$$
K Z=-k(n I-\mathbb{1}), \quad k=\frac{n+\sqrt{n^{2}+4 n}}{n\left(2+n+\sqrt{n^{2}+4 n}\right)} .
$$

The matrix $W$ can be interpreted as the incident matrix of a complete digraph $\mathcal{G}=(V, E)$, with $\operatorname{card}(V)=n$, where edges between vertices represent communication between vehicles. The matrix $Z$ is the incident matrix of a directed tree in the graph $\mathcal{G}$. Each pair of edges $(i, j)$ and $(j, i), i<j$ represents a quantized communication channel between vehicle $i$ and vehicle $j$.

\footnotetext{
3 The matrix $\mathbb{1}$ represent the $n \times n$ unit matrix.
} 


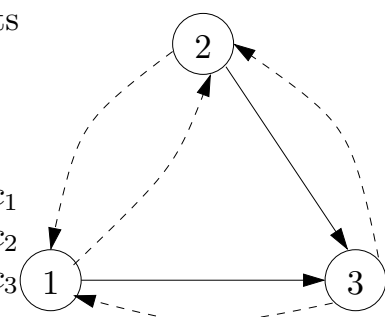

Topology 1

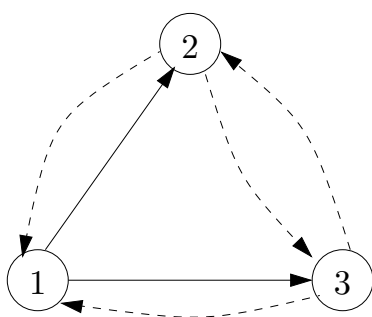

Topology 2

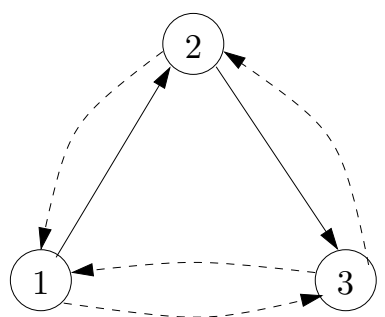

Topology 3

Fig. 2. Three different communication topologies for $n=3$. Solid lines denote uniformly quantized communication channels, and dashed lines logarithmically quantized communication channels.

\subsection{Uniform quantization}

Proposition 7. The feedback

$$
u_{i}=-k(n-1) x_{i}+k \sum_{j=1}^{n} q_{u}\left(x_{j}\right),
$$

with $k$ as in (12), solves the rendezvous problem.

Proof. Similar to the proof of Proposition 8.

\subsection{Uniform-logarithm quantization}

Since the logarithmic quantized channels are more efficient, because less bits need to be transmitted compared with uniform quantized channels, we would like to have a communication topology with as many link as possible. Let $n=3$, we consider topologies where the digraph $\mathcal{G}$ has a tree representing uniformly quantized channels and the remaining edges representing logarithmically quantized channels. Since we have tree vehicles the number of possible directed trees, up to a re-labeling of the vertices are three as shown in Figure 2.

Proposition 8. Let us define the following vectors corresponding to the three different topologies shown in Figure 2,

$$
\begin{aligned}
c_{1}^{1} & =\left(q_{\ell}\left(q_{u}\left(x_{1}\right)-x_{3}\right), q_{\ell}\left(q_{u}\left(x_{2}\right)-x_{3}\right)\right) \\
c_{2}^{1} & =\left(q_{\ell}\left(q_{u}\left(x_{1}\right)-x_{3}\right), q_{\ell}\left(q_{u}\left(x_{2}\right)-x_{3}\right)\right) \\
c_{3}^{1} & =\left(q_{u}\left(x_{1}\right), q_{u}\left(x_{2}\right)\right) \\
c_{1}^{2} & =\left(q_{\ell}\left(q_{u}\left(x_{1}\right)-x_{2}\right), q_{\ell}\left(q_{u}\left(x_{1}\right)-x_{3}\right)\right) \\
c_{2}^{2} & \left.=\left(q_{u}\left(x_{1}\right)-x_{2}\right), q_{\ell}\left(q_{u}\left(x_{1}\right)-x_{3}\right)\right) \\
c_{3}^{2} & =\left(q_{u}\left(x_{1}\right), q_{\ell}\left(q_{u}\left(x_{1}\right)-x_{2}\right)\right) \\
c_{1}^{3} & =\left(q_{\ell}\left(q_{u}\left(x_{1}\right)-x_{2}\right), q_{\ell}\left(q_{u}\left(x_{2}\right)-x_{3}\right)\right) \\
c_{2}^{3} & =\left(q_{u}\left(x_{1}\right), q_{\ell}\left(q_{u}\left(x_{2}\right)-x_{3}\right)\right) \\
c_{3}^{3} & =\left(q_{\ell}\left(q_{u}\left(x_{1}\right)-x_{2}\right), q_{u}\left(x_{2}\right)\right) .
\end{aligned}
$$

The feedback control laws

$u_{i}^{j}(t)=-k_{i, 1}^{j} c_{i, 1}^{j}-k_{i, 2}^{j} c_{i, 2}^{j} \forall i=1, \ldots, 3 \forall j=1, \ldots, 3$ with $\left[k_{i, 1}^{j}, k_{i, 1}^{j}\right]$, for $i=1,2,3$ and $j=1,2,3$, ith row of the matrix $K$ defined in (12) with $n=3$, solve the rendezvous problem.

Proof. Assume the communication topology is the first one (see Figure 2). We prove the statement for such case, the other can be proved in a similar way. In this case

$$
K=k\left(\begin{array}{cc}
2 & -1 \\
-1 & 2 \\
-1 & -1
\end{array}\right)
$$

We introduce the Lyapunov function $\mathcal{V}\left(z_{i, j}\right)=$ $\left|z_{i, j}\right|$, with $z_{i, j} \in\left\{z_{1,3}, z_{2,3}\right\}$ Let $\Delta \mathcal{V}\left(z_{i, j}\right)=$ $\mathcal{V}\left(z_{i, j}^{+}\right)-\mathcal{V}\left(z_{i, j}\right)$, then we have

$$
\begin{aligned}
\Delta \mathcal{V}\left(z_{1,3}\right) & \leq-3 k\left|z_{1,3}\right|+2 k \delta^{2}+2 k \delta\left|z_{1,3}\right|+k \delta^{2} \\
& +k \delta\left|z_{2,3}\right|+3 k \delta \\
\Delta \mathcal{V}\left(z_{2,3}\right) & \leq-3 k\left|z_{2,3}\right|+2 k \delta^{2}+2 k \delta\left|z_{2,3}\right|+k \delta^{2} \\
& +k \delta\left|z_{1,3}\right|+3 k \delta .
\end{aligned}
$$

Thus we have $\Delta \mathcal{V}\left(z_{1,3}\right)<0$ and $\Delta \mathcal{V}\left(z_{2,3}\right)<$ if

$$
\begin{aligned}
&\left|z_{1,3}\right|>\frac{3 \delta(1+\delta)+\delta\left|z_{2,3}\right|}{3-2 \delta} \\
&\left|z_{2,3}\right|>\frac{3 \delta(1+\delta)+\delta\left|z_{1,3}\right|}{3-2 \delta} .
\end{aligned}
$$

For any $\epsilon>0$ there exists $\delta=-1 / 2-\epsilon / 2+$ $\sqrt{1+6 \epsilon+\epsilon^{2}} / 2$ such that $z_{1,3}$ and $z_{2,3}$ asymptotically converges to the ball $\mathcal{B}_{\epsilon}$. Thus the feedback (13) solve the rendezvous problem.

\section{SIMULATION RESULTS}

A simulation study have been done for the threevehicle case. In Figure 3 with are shown the trajectories of the three vehicles for the three different communication topologies of Figure 2. In dotted line is shown the trajectory of the vehicles when the communication is without quantization (perfect). In solid line is shown the trajectories of the vehicles communicating using topology 1 , in dashed line the trajectories when topology 2 is used and in dashed-dotted the trajectories when topology 3 is used. We can notice the three vehicles rendezvous (at the point marked with a 


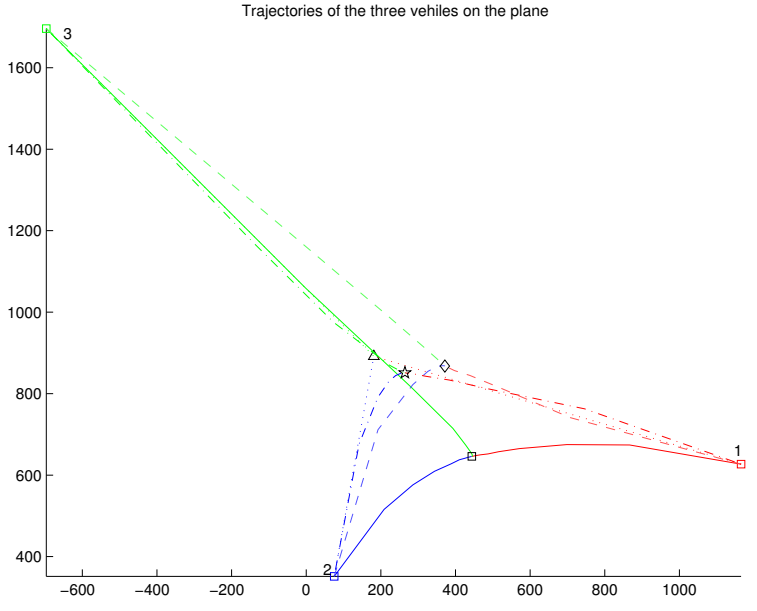

Fig. 3. Trajectories for three vehicles for the three different topologies of figure 2 . In the simulations we assumed the uniform quantization error equal to zero.

square, diamond and star), but trajectories are very different depending on the topology. If we consider the time evolution of $z_{1,2}=x_{1}-x_{2}$ and $z_{2,3}=x_{2}-x_{3}$ and shown in Figure (4(a)) (in Figure $(4(\mathrm{~b}))$ are shown similar time evolutions for the relative distances in the y-coordinate), we can notice that vehicles communicating using the topology 1 (cf., Figure 2) rendezvous slowly than when communicating using the other two topologies. This behavior can be explained considering that the third vehicle knows with higher accuracy the position of the two vehicles $V_{1}, V_{2}$ while these two last have a very rough information of their relative distance to the vehicle $V_{3}$, due to the logarithmically quantized communication. This results in slower performance, compare to the remaining topologies.

\section{CONCLUSIONS}

In this paper we have considered the "multivehicle" rendezvous problem under quantized communication topologies. In particular results have been derived for two and three vehicles systems for different topologies and various configurations of uniform and logarithmic quantizers. Some simulation results showing the behavior of the different topologies have been studied in order to verify the results. The trajectories followed by the vehicles seem to depend upon the communication topology used.

\section{REFERENCES}

Beghi, A. and D. D'Alessandro (1998). Discretetime optimal control with control-dependent noise and generalized Riccati difference equation. Automatica 34(8), 1031-1034.
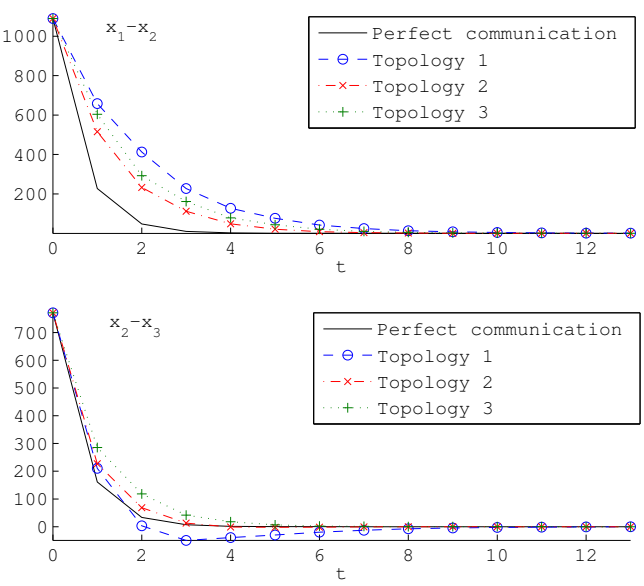

(a) Differences $x_{1}(t)-x_{2}(t)$ and $x_{2}(t)-x_{3}(t)$ for the three vehicles corresponding to the trajectories of Figure 3.
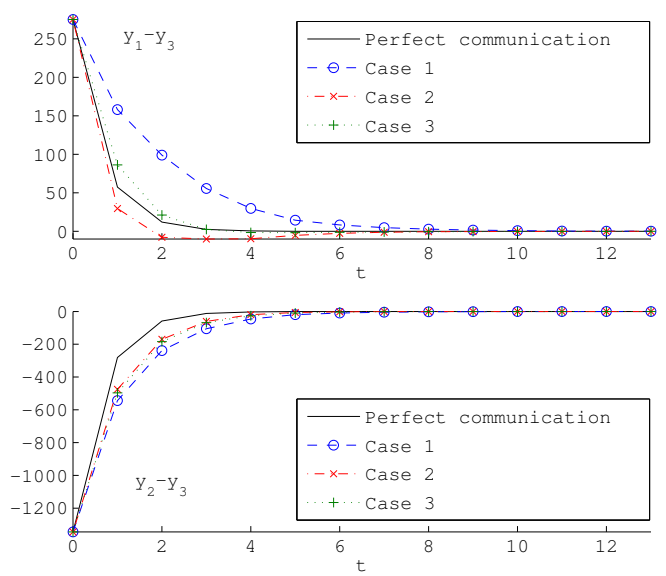

(b) Differences $y_{1}(t)-y_{2}(t)$ and $y_{2}(t)-y_{3}(t)$ for the three vehicles corresponding to the trajectories of Figure 3.

Fig. 4. Performance comparison of the difference communication topologies.

de Sousa, J. B. and F. L. Pereira (2002). On coordinated control strategies for networked dynamic control systems: an application to AUV's. In: Proc. MTNS.

Fagnani, F. and S. Zampieri (2003). Stability analysis and synthesis for scalar linear systems with quantized feedback. IEEE Trans. Automat. Control 48(9), 1569-1584.

Lin, J., A. S. Morse and B. D. O. Anderson (2003). The multi-agent rendezvous problem. In: Proc. IEEE CDC.

Saber, R. O. and R. M. Murray (2003). Flocking with obstacle avoidance: cooperation with limited communication in mobile networks.. In: Proc. IEEE CDC.

Speranzon, A. and K. H. Johansson (2003). On some communication schemes for distributed pursuit-evasion games. In: Proc. IEEE CDC.

Varaiya, P. (1993). Smart cars on smart roads: Problems of control. IEEE Trans. Automat. Control 38(2), 195-207. 